\title{
Capture of dengue virus type 3 using anionic polymer-coated magnetic beads
}

\author{
AKIKAZU SAKUDO ${ }^{1,2}$, PROMSIN MASRINOUL ${ }^{1}$, YASUHARU TANAKA $^{2}$ and KAZUYOSHI IKUTA ${ }^{1}$ \\ ${ }^{1}$ Department of Virology, Center for Infectious Disease Control, Research Institute for Microbial Diseases, Osaka University, \\ Yamadaoka, Suita, Osaka 565-0871; ${ }^{2}$ Laboratory of Biometabolic Chemistry, School of Health Sciences, \\ Faculty of Medicine, University of the Ryukyus, Nishihara, Okinawa 903-0215, Japan
}

Received March 10, 2011; Accepted April 26, 2011

DOI: $10.3892 / \mathrm{ijmm} .2011 .733$

\begin{abstract}
Dengue virus (DENV) is a mosquito-borne virus and can be transmitted to humans by mosquito vectors. Although surveillance of dengue virus-infected mosquitoes is the most effective way of controlling DENV infections, detection of DENVs in mosquitoes is limited by the low sensitivity of available detection methods. We here report a method for capturing DENV type 3 (DENV-3) from mosquito cells using magnetic beads coated with an anionic polymer, poly(methyl vinyl ether-maleic anhydrate). The beads were incubated with cell culture medium of DENV-3-infected mosquito cells, then separated from the supernatant by applying a magnetic field and washed. Adsorption of DENV-3 on the beads was confirmed by reverse transcription-polymerase chain reaction, which detected the presence of DENV-3 genomic RNA on the beads, and Western blotting, which determined the major DENV-3 envelope protein on the beads. Therefore, this capture method may enable an improvement in DENV-3 detection.
\end{abstract}

\section{Introduction}

Dengue virus (DENV) is a mosquito-borne, plus-strand RNA virus with an enveloped icosahedral nucleocapsid, a member of the genus Flavivirus, family Flaviviridae, and the etiological agent of infectious disease affecting almost 50 million people worldwide (1). DENV infection can lead to dengue fever (DF), dengue hemorrhagic fever (DHF), and dengue shock syndrome (DSS). DHF and DSS are potentially lethal: the risk of developing them is correlated with infection by one of the four DENV serotypes (DENV-1 to -4) and to having antibodies to

Correspondence to: Dr Akikazu Sakudo, Laboratory of Biometabolic Chemistry, School of Health Sciences, Faculty of Medicine, University of the Ryukyus, 207 Uehara, Nishihara, Okinawa 903-0215, Japan

E-mail: sakudo@med.u-ryukyu.ac.jp

Key words: anionic polymer, virus concentration, dengue virus, virus capture another DENV serotype from a previous infection (2). Unfortunately, no specific treatment or vaccine for DENV is available.

South-East Asia is one of the most seriously DENV-affected areas $(1,3)$. DENV is mostly found in urban and semi-urban areas in tropical and sub-tropical regions reflecting the geographic distribution of its mosquito vectors, in particular Aedes aegypti and Aedes albopictus. The range of these mosquitoes has been expanding and, thereby, enabling spread of DENV (1). Therefore, control of DENV-infected mosquitoes is the most effective approach for preventing DENV. However, the distribution of DENV-infected mosquitoes remains unclear because of the low sensitivity of methods for DENV detection in mosquitoes. Therefore, a sensitive method for detection of DENV is needed. To develop such a method, we chose DENV type 3 (DENV-3) for this study, because of a 1998 outbreak of DENV-3 and recent major DENV outbreaks in Taiwan (4-6). This is of concern to us because Taiwan is only $127 \mathrm{~km}$ from Yonaguni Island, the southernmost island of the Ryukyu archipelago of Japan (7).

We report here the use of magnetic beads coated with an anionic polymer for the capture of DENV-3 from the medium of a mosquito cell culture infected with DENV-3.

\section{Materials and methods}

Reagents. Unless otherwise specified, chemical reagents were obtained from Sigma (St. Louis, MO) or Wako Pure Chemical Industries (Osaka, Japan). Anionic polymer-coated magnetic beads, Viro-Adembeads, were obtained from Ademtech (Pessac, France). These are $300 \mathrm{~nm}$-diameter paramagnetic beads coated with the anionic polymer poly(methyl vinyl ether-maleic anhydrate), [poly(MVE-MA)], to which the surface of some virus particles bind (Flavigny E, et al, 104th General Meeting, New Orleans: abs. 166, 2004).

Cell culture and virus. A C6/36 cell culture (derived from Aedes albopictus) was maintained in Leibovitz L-15 medium containing $0.3 \%$ tryptose phosphate broth (TPB) and $10 \%$ fetal calf serum (FCS). A laboratory DENV strain (DENV-3, 80-2) isolated from a DF patient in China (GenBank Accession no. AF317645) was used to infect C6/36 cell cultures. C6/36 cells were infected with DENV-3 at a multiplicity of infection 


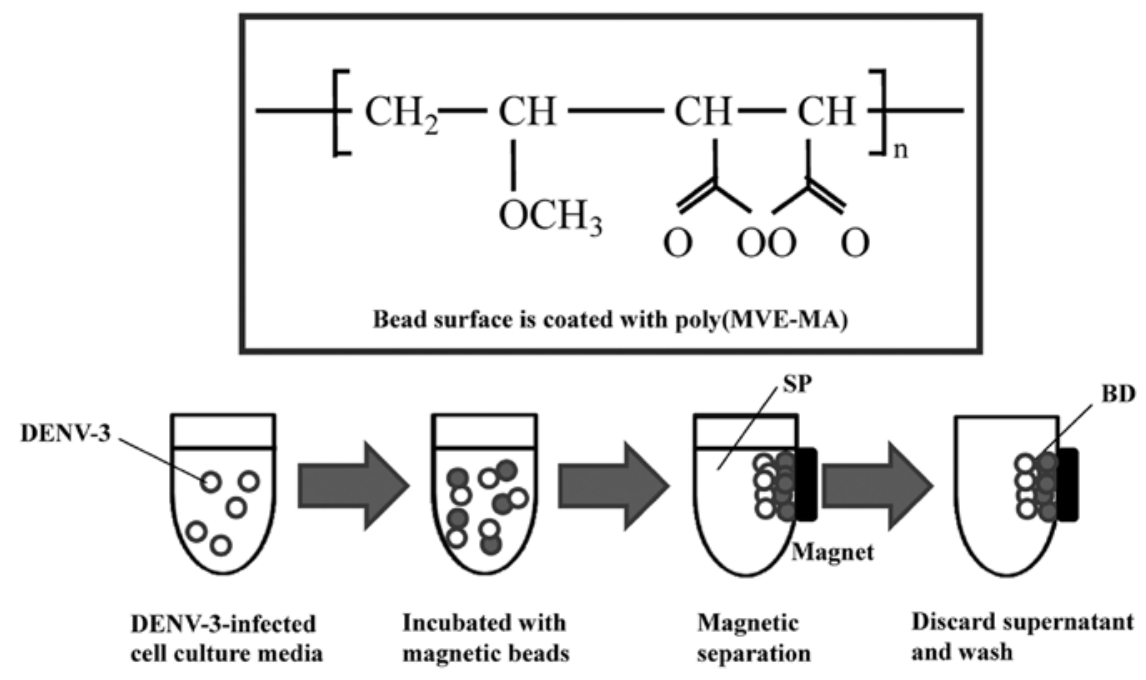

Figure 1. DENV-3 capture by anionic polymer-coated magnetic beads. Schematic of the protocol for capture of DENV-3 from the medium of C6/36 mosquito cells infected by DENV-3, and separation of the bead (BD) and supernatant (SP) fractions. A sample of the medium with the same volume as the BD fraction was also analyzed as the total sample fraction (TL). Modified from Figs. 1 and 2 in Sakudo et al (9) with permission from Elsevier.

of 0.1 in Leibovitz L-15 medium containing $0.3 \%$ TPB and $2 \% \mathrm{FCS}$, incubated for 3 days at $28^{\circ} \mathrm{C}$, and the medium was then collected and used for virus capture studies.

$D E N V$-3 capture. Virus capture was performed using the manufacturer's instructions (Ademtech). Briefly, $40 \mu 1$ ViroAdembeads were washed twice with binding buffer and twice with phosphate-buffered saline (PBS). A $40 \mu 1$ sample of medium from an uninfected (Mock) or DENV-3-infected cell culture and $360 \mu \mathrm{l}$ PBS were added to the washed beads and the tube was incubated for $20 \mathrm{~min}$ at room temperature (Fig. 1). The tubes containing the mixtures were set in a magnetic field for 1 min using an Adem-Mag SV magnetic device (Ademtech). After magnetic separation, the supernatant was removed, and the beads were washed three times with PBS and then resuspended in $40 \mu \mathrm{l}$ PBS. This produced two fractions: a bead fraction (designated BD) and a supernatant fraction (designated SP). A total sample fraction (i.e., the cell culture medium originally mixed with the beads) (designated TL) was also included. A $40 \mu 1$ sample of each fraction was used for RT-PCR and Western blot analyses.

Western blotting. Each fraction was solubilized in an equal volume of $2 \mathrm{X}$ sodium dodecyl sulphate (SDS) gel-loading buffer [ $90 \mathrm{mM}$ Tris- $\mathrm{HCl}$ (pH 6.8), 10\% mercaptoethanol, 2\% SDS, $0.02 \%$ bromophenol blue, and $20 \%$ glycerol], boiled for $5 \mathrm{~min}$, followed by separation on an $8 \%$ polyacrylamide gel by SDS-polyacrylamide gel electrophoresis (PAGE). The proteins were then electroblotted onto a polyvinylidene difluoride (PVDF) membrane (Hybond-P; GE Healthcare, Piscataway, $\mathrm{NJ}$ ) for $60 \mathrm{~min}$ at $15 \mathrm{~V}$. After protein transfer, the membranes were treated with 5\% skim milk for $1 \mathrm{~h}$ at room temperature, and then incubated with anti-DENV-3 envelope (Env) antibody D1-11(3) (GeneTex, Inc., Irvine, CA) in PBS containing 0.1\% Tween-20 (PBS-T) and 0.5\% skim milk for $1 \mathrm{~h}$ at room temperature. After three washes with PBS-T, the membranes were incubated in horseradish peroxidase (HRP)-conjugated antimouse IgG (Jackson ImmunoResearch Laboratories, Inc.,
West Grove, PA, USA) in PBS-T and 0.5\% skim milk for $1 \mathrm{~h}$ at room temperature. After three washes with PBS-T, the proteins were detected using an enhanced chemiluminescence detection kit (GE Healthcare).

Reverse transcription (RT)-PCR. Viral RNA from each fraction was extracted with a QIAamp Viral RNA Mini kit (Qiagen, Hilden, Germany) according to the manufacturer's instructions. RT reactions were then carried out using the ThermoScript RT-PCR System (Invitrogen, Grand Island, NY) with random primers. After incubation at $25^{\circ} \mathrm{C}$ for $10 \mathrm{~min}$, viral RNA was reverse-transcribed at $65^{\circ} \mathrm{C}$ for $50 \mathrm{~min}$, followed by denaturation of the reverse transcriptase at $85^{\circ} \mathrm{C}$ for $5 \mathrm{~min}$. The resultant cDNA was amplified by PCR in a reaction mixture containing primers, Ex Taq (Takara Bio Inc., Otsu, Japan), and Ex Taq buffer under conditions of 35 cycles at $94^{\circ} \mathrm{C}$ for $1 \mathrm{~min}, 60^{\circ} \mathrm{C}$ for $1 \mathrm{~min}$ and $72^{\circ} \mathrm{C}$ for $1 \mathrm{~min}$. The primers were: DENV-3-F, 5'-accgtgtgtcaactggatca-3', and DENV-3-R, 5'-gtcatggatggggt aaccag-3'. The amplified DNA fragments were purified and analyzed by DNA sequencing (ABI-PRISM 3100 Genetic Analyzer, Applied Biosystems, Foster City, CA) to verify the amplified product.

Real-time RT-PCR. The cDNAs produced in the RT reactions above were also analyzed by real-time PCR quantitative PCR (QPCR). For QPCR, a Brilliant SYBR-Green QPCR Master Mix was used according to the manufacturer's instructions (Stratagene, La Jolla, CA). Briefly, the QPCR components included Brilliant QPCR Master mix, reverse-transcribed cDNA, and the forward and reverse target gene primers: DENV-3real-F, 5'-acttcctccectgcgatac-3', and DENV-3real-R, 5'-ggtctgatttccatgccatac-3'. QPCR was performed in the Mx3000P real-time QPCR system (Stratagene) under the following conditions: denaturation at $95^{\circ} \mathrm{C}$ for $10 \mathrm{~min}$ followed by 40 cycles of denaturation $\left(95^{\circ} \mathrm{C}\right.$ for $\left.30 \mathrm{sec}\right)$, annealing $\left(58^{\circ} \mathrm{C}\right.$ for $60 \mathrm{sec})$, and extension $\left(72^{\circ} \mathrm{C}\right.$ for $\left.30 \mathrm{sec}\right)$. Each reaction was performed in triplicate. The results were analyzed using the Mx3000P system software. The relative expression ratio of 


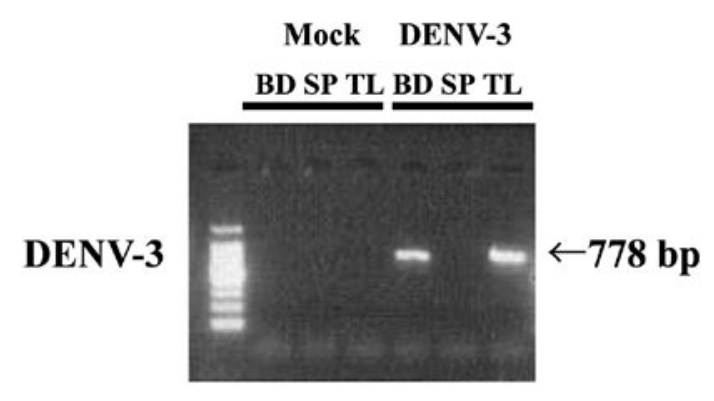

Figure 2. Detection of viral RNA from DENV-3 captured on anionic polymer-coated magnetic beads. Culture media of DENV-3-infected (DENV-3) and uninfected (Mock) C6/36 cells were mixed with anionic polymer-coated magnetic beads and separated into BD and SP fractions. The total fraction (TL) was a sample of the cell culture medium originally mixed with the beads. Viral RNA was extracted from each fraction using a QIAamp Viral RNA Mini kit (Qiagen, Hilden, Germany) and analyzed by RT-PCR. The PCR primers were specific for a 778 bp fragment of the DENV-3 strain 80-2 genome. A $100 \mathrm{bp}$ ladder marker is shown on the left.

each sample was calculated by a mathematical model based on the amplification efficiency. PCR specificity was verified by dissociation curve analysis of the amplified DNA fragments.

\section{Results and Discussion}

To investigate whether Viro-Adembeads could be used to capture DENV-3, medium from DENV-3-infected C6/36 mosquito cells was mixed with the anionic polymer-coated magnetic beads. The mixture was magnetically separated and bead (BD), supernatant (SP) and total (TL) fractions were prepared (Fig. 1). Medium from mock-infected cells was used to prepare control fractions. The fractions were analyzed by RT-PCR, Western blotting, and real-time RT-PCR to determine the extent of DENV-3 capture by the beads.

To examine DENV-3 capture by the beads, RT-PCR was used to look for DENV-3 RNA in the BD, SP and TL fractions (Fig. 2). Using primers specific for a 778-bp sequence in the DENV-3 strain 80-2 genome, a single 778-bp band was found in the BD and TL fractions, but not in the SP fraction. The 778-bp band was confirmed by two independent DNA sequence analyses to be the DENV-3 strain 80-2 genomic sequence (86 and $97 \%$ identity to GenBank accession no.AF317645 within the sequenced region). These results indicate that the BD fraction contained DENV-3 genomic RNA and, therefore, that the beads had captured DENV-3 viruses.

Western blotting of the BD, SP and TL fractions from DENV-3-infected C6/36 cells using anti-DENV-3 Env protein antibody showed a $61 \mathrm{kDa}$ band in the BD and TL fractions, but not the SP fraction (Fig. 3). The $61 \mathrm{kDa}$ size is in agreement with that calculated from the deduced amino acid sequence of the DENV-3 Env protein. These results, that DENV-3 was detected in the BD and TL fractions but not in the SP fraction by Western blotting, were consistent with the results from RT-PCR analysis, indicating that DENV-3 was captured by the anionic polymer-coated magnetic beads.

The amount of DENV-3 RNA in the BD, SP and TL fractions was measured by real-time RT-PCR, relative to that in the control (a TL sample with highest value was taken as $100 \%)$. For the DENV-3-infected cell culture medium, the

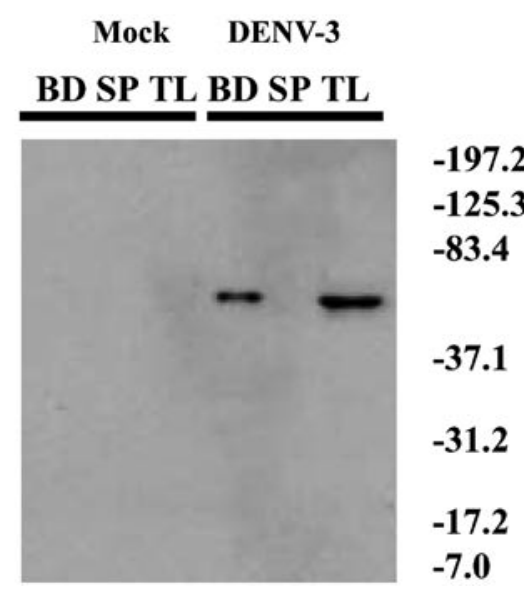

Figure 3. Detection of the DENV-3 Env protein captured on anionic polymercoated magnetic beads. BD, SP and TL fractions from DENV-3-infected and uninfected (Mock) C6/36 cells were solubilized with SDS and analyzed by Western blotting with anti-DENV-3 antibody against the DENV-3 Env protein $(61 \mathrm{kDa})$. Molecular weight markers $(\mathrm{kDa})$ are shown on the right.

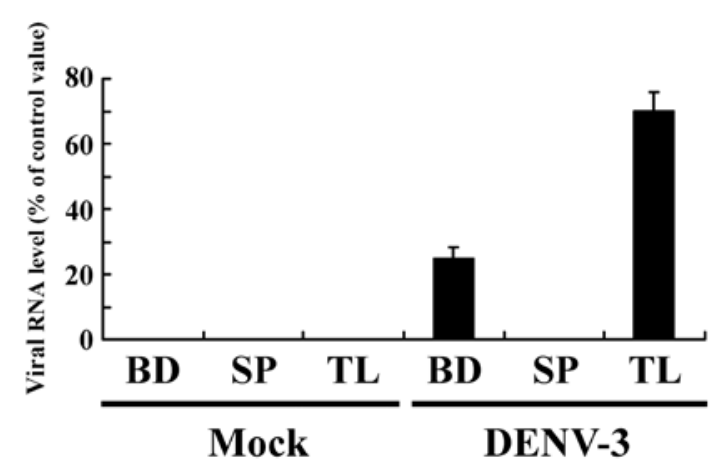

Figure 4. Quantitative analysis of viral RNA in DENV-3 captured on anionic polymer-coated magnetic beads. DENV-3 in $400 \mu 1$ diluted culture media from DENV-3-infected and uninfected (Mock) C6/36 cells was captured on anionic polymer-coated magnetic beads and separated into BD, SP, and TL fractions. These fractions were analyzed by real-time RT-PCR for the relative amount of DENV-3 genomic RNA.

amount of viral RNA in the BD fraction was about one third of that in the TL fraction (Fig. 4). The specificity of these PCR reactions was confirmed by dissociation curve analysis of the reaction products (data not shown). These results showed that DENV-3 in cell culture medium could be captured by the magnetic beads. However, a significant fraction of DENV-3 in the culture medium of virus-infected cells was lost during the capture procedure using anionic polymer-coated magnetic beads. This may have been due to serum components in the cell culture medium, such as albumin, binding to the magnetic beads and inhibiting virus capture (8).

This and other studies have shown that magnetic beads coated with the anionic polymer poly(MVE-MA) can be used to capture various viruses, such as DENV-3, avian and human influenza virus, respiratory syncytial virus (RSV), vaccinia virus, borna disease virus, and human herpesvirus 8 (8-12). In these studies, the anionic polymer-coated magnetic beads have been shown to facilitate detection of viruses and to be compatible with various analytical methods, such as PCR, ELISA, immunochromato-graphy and Western blotting. 
Although in previous studies the beads aggregated after incubation with influenza A and B virus- and RSV-infected samples $(8,9)$, aggregation was not observed in this study of DENV-3. Therefore, aggregation did not appear to be involved in the capture of DENV-3. Our preliminary studies have shown that hepatitis B virus (an enveloped DNA virus) was not captured by poly(MVE-MA)-coated magnetic beads (Sakudo, unpublished data). Since DENV-3 and hepatitis B virus are both enveloped viruses, it appears that not all enveloped viruses can bind to poly(MVE-MA)-coated magnetic beads. Therefore, information on the charge density and steric spatial organization of the anionic poly(MVE-MA) coating on magnetic beads would be useful in trying to understand the interaction between the beads and viruses. In addition, as described above, attention should be paid to non-specific binding of other media components, like albumin, that might affect the charge density and surface chemistry of the poly(MVE-MA) coating and change non-specific virus binding. Regarding this possibility, a recent preliminary report has shown that the spatial organization of the poly(MVE-MA) polymer affected binding activity.

In conclusion, we have demonstrated that magnetic beads coated with an anionic polymer are useful for the capture of DENV-3. DENV-3 capture by the coated beads was confirmed by showing that the beads fraction (BD) contained DENV-3 genomic RNA and Env proteins by RT-PCR, Western blotting, and real-time RT-PCR. Therefore, this method can be used in combination with conventional means of detection and may increase the sensitivity of virus detection for DENV-3 diagnosis. The applicability of this method to other DENV serotypes is promising. In addition, the adaptability of this method to different types of viruses is currently under investigation.

\section{Acknowledgements}

The authors are grateful to Dr Yoshinobu Okuno (Research Foundation for Microbial Diseases of Osaka University) for providing DENV-3. This study was partly supported by the Japan Science and Technology Agency (JST)/Japan International
Cooperation Agency (JICA), Science and Technology Research Partnership for Sustainable Development (SATREPS), the Ministry of Education, Culture, Sports, Science and Technology (MEXT), and the Ministry of Economy, Trade, and Industry (METI).

\section{References}

1. World Health Organization: Dengue and Dengue Haemorrhagic Fever. Fact sheet, WHO, Geneva, 2009. Available from: http:// www.who.int/mediacentre/factsheets/fs117/en/.

2. Morens DM: Antibody-dependent enhancement of infection and the pathogenesis of viral disease. Clin Infect Dis 19: 500-512, 1994.

3. Lindback H, Lindback J, Tegnell A, Janzon R, Vene S and Ekdahl K: Dengue fever in travelers to the tropics, 1998 and 1999. Emerg Infect Dis 9: 438-442, 2003.

4. Harris E, Roberts TG, Smith L, et al: Typing of dengue viruses in clinical specimens and mosquitoes by single-tube multiplex reverse transcriptase PCR. J Clin Microbiol 36: 2634-2639, 1998.

5. Huang JH, Liao TL, Chang SF, et al: Laboratory-based dengue surveillance in Taiwan, 2005: a molecular epidemiologic study. Am J Trop Med Hyg 77: 903-909, 2007.

6. World Health Organization: Dengue Hemorrhagic Fever: Diagnosis, Treatment Prevention and Control. 2nd edition. WHO, Geneva, 1997.

7. Tanaka K, Mizusawa K and Saugstad ES: A revision of the adult and larval mosquitoes of Japan (including the Ryukyu Archipelago and the Ogasawara Islands) and Korea (Diptera: Culicidae). Contrib Am Entomol Inst 16: 1-987, 1979.

8. Sakudo A, Baba K, Tsukamoto M, et al: Anionic polymer, poly(methyl vinyl ether-maleic anhydride)-coated beads-based capture of human influenza A and B virus. Bioorg Med Chem 17: 752-757, 2009.

9. Sakudo A, Baba K, Tsukamoto $\mathrm{M}$ and Ikuta K: Use of anionic polymer, poly(methyl vinyl ether-maleic anhydride)-coated beads for capture of respiratory syncytial virus. Bioorg Med Chem Lett 19: 4488-4491, 2009.

10. Sakudo A and Ikuta K: Efficient capture of infectious H5 avian influenza virus utilizing magnetic beads coated with anionic polymer. Biochem Biophys Res Commun 377: 85-88, 2008.

11. Hatano B, Kojima A, Sata T and Katano H: Virus detection using Viro-Adembeads, a rapid capture system for viruses, and plaque assay in intentionally virus-contaminated beverages. Jpn J Infect Dis 63: 52-54, 2010.

12. Sakudo A, Tanaka Y and Ikuta K: Capture of infectious borna disease virus using anionic polymer-coated magnetic beads. Neurosci Lett 494: 237-239, 2011. 